\title{
Problem on unresolved resonance data in recent nuclear data libraries
}

\author{
C. Konno ${ }^{\text {a }}$ T. Nakagawa, K. Ochiai, S. Sato, and M. Yamauchi \\ Japan Atomic Energy Agency, Ibaraki 319-1195, Japan
}

\begin{abstract}
It is found out that self-shielding correction for unresolved resonance data of many nuclei in JENDL-3.3 and ENDF/B-VII.0 is too large around the upper boundary energy of the unresolved resonance region. It is confirmed that this issue can be solved by increasing the upper boundary energy of the unresolved resonance region. In evaluation for unresolved resonance data, the upper boundary energy of the unresolved resonance region should be set to be high enough by considering self-shielding correction.
\end{abstract}

\section{Introduction}

Nuclear data libraries have unresolved resonance data. However these unresolved resonance data were not effectively used in neutronics calculations since calculation codes could not always deal with unresolved resonance data so far.

At the international conference on nuclear data for science and technology held in 2001, we pointed out [1] that the leakage neutron spectrum from a niobium sphere of $0.5 \mathrm{~m}$ in radius with a $20 \mathrm{MeV}$ neutron in the center, which was calculated with ANISN [2], MCNP4C [3] (this version can treat unresolved resonance data) and JENDL-3.3 [4], had a large strange bump around $100 \mathrm{keV}$ as shown in figure 1 , which originated from self-shielding correction for the unresolved resonance data. The following points for this phenomenon are investigated in details in this paper.

What in unresolved resonance data causes this phenomenon?

How about other nuclei in JENDL-3.3?

How about other nuclear data libraries?

\section{Self-shielding correction for unresolved resonance region of ${ }^{93} \mathrm{Nb}$ in JENDL-3.3}

The unresolved resonance data of ${ }^{93} \mathrm{Nb}$ in JENDL-3.3 are considered to have some problems. In order to check the cross section data of ${ }^{93} \mathrm{Nb}$ in JENDL-3.3, the following multigroup libraries (VITAMIN-J group structure [5]) of pure ${ }^{93} \mathrm{Nb}$ in JENDL-3.3 were produced with self-shielding correction in the resolved resonance region;

1) multigroup library with self-shielding correction in the unresolved resonance region,

2) multigroup library without self-shielding correction in the unresolved resonance region.

The first multigroup library was generated with the TRANSX code [6] from the MATXSLIB-J33 file [7]. The second multigroup library were made with the TRANSX code from a

${ }^{a}$ Presenting author, e-mail: konno.chikara@jaea.go.jp

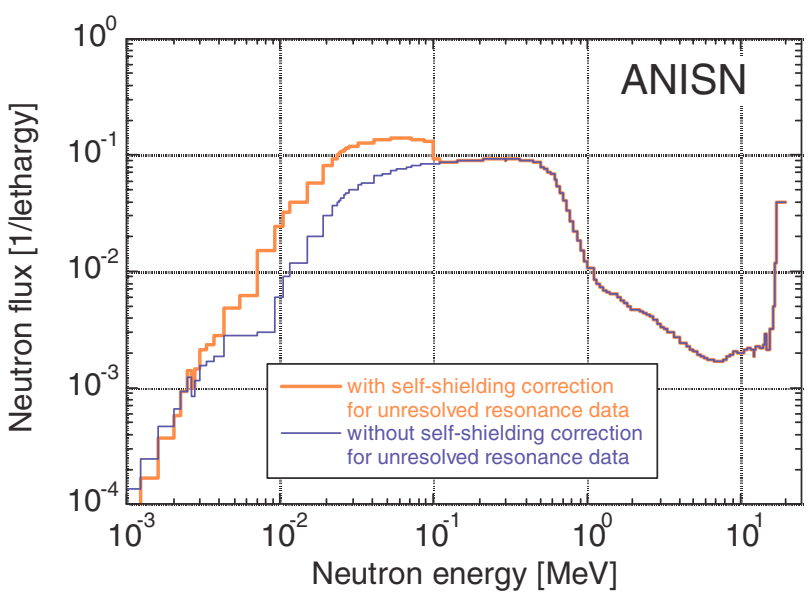

(a) ANISN calculation

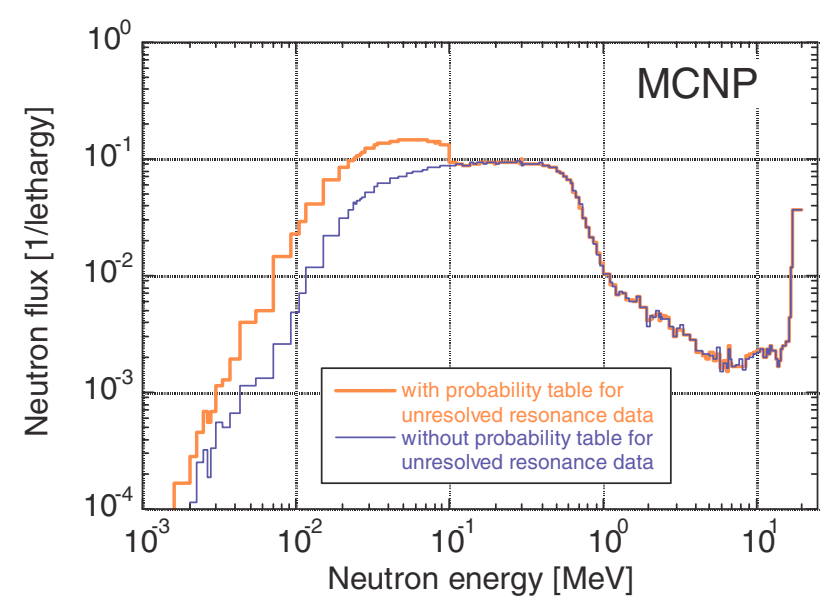

(b) MCNP calculation

Fig. 1. Calculated leakage neutron spectra from a niobium sphere of $0.5 \mathrm{~m}$ in radius with a $20 \mathrm{MeV}$ neutron in the center by using (a) ANISN and (b) MCNP.

MATXS file produced from JENDL-3.3 with the NJOY99.67 code [8] and patch [7] for JENDL-3.3 in the same condition as MATXSLIB-J33 except for skipping self-shielding correction for unresolved resonance data. These multigroup 


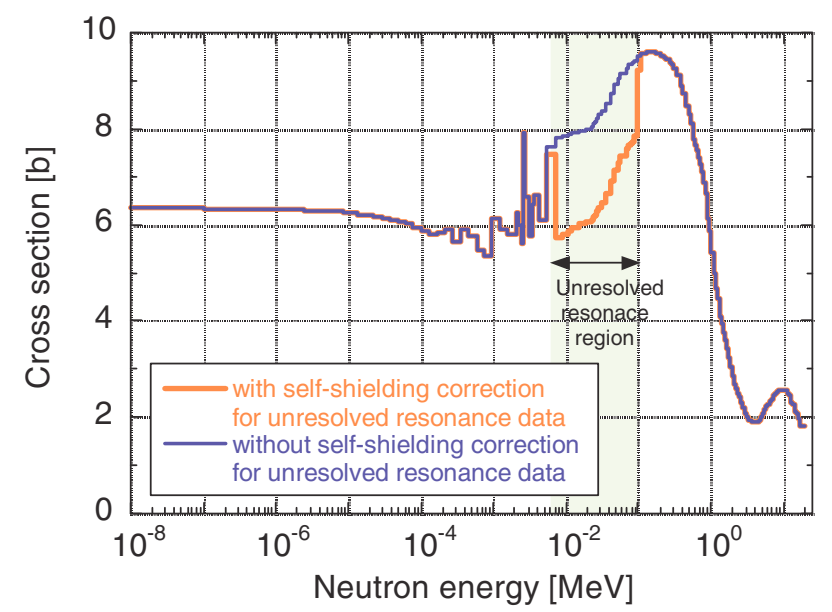

Fig. 2. Self-shielding corrected elastic scattering cross section of ${ }^{93} \mathrm{Nb}$ in JENDL-3.3.

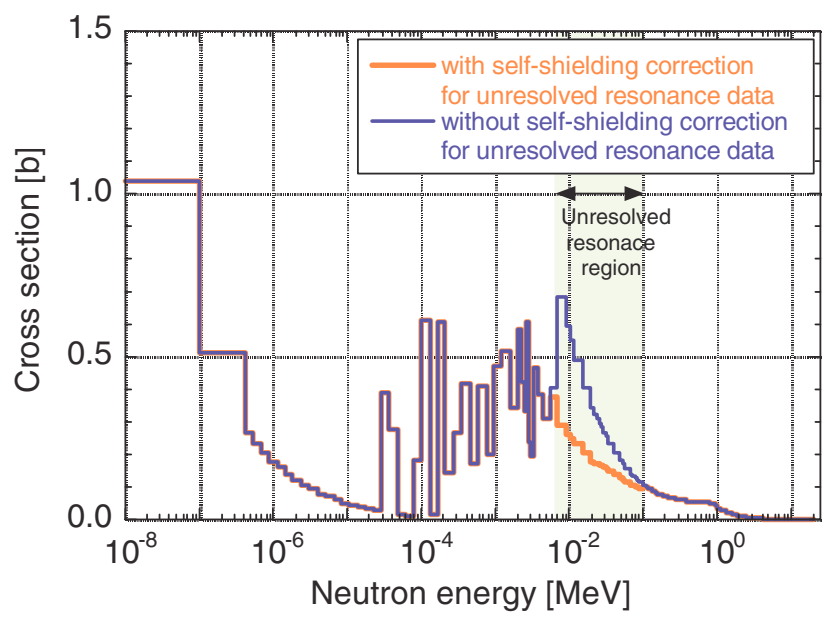

Fig. 3. Self-shielding corrected $(\mathrm{n}, \gamma)$ reaction cross section of ${ }^{93} \mathrm{Nb}$ in JENDL-3.3.

libraries include response data such as elastic scattering and $(\mathrm{n}, \gamma)$ reaction cross sections. Since the unresolved resonance data are given for elastic scattering and $(n, \gamma)$ reaction, selfshielding correction for the elastic scattering and $(\mathrm{n}, \gamma)$ cross section data was investigated in the unresolved resonance region. Figures 2 and 3 plot the elastic scattering and (n, $\gamma)$ reaction cross section data deduced from the above multigroup libraries, respectively. It is found out that the elastic scattering cross sections of ${ }^{93} \mathrm{Nb}$ with self-shielding correction have a large bump at the upper boundary energy [100 keV] of the unresolved resonance region. The self-shielding correction for the elastic scattering cross section data in the unresolved resonance region is too large around the upper energy of the unresolved resonance region, while that for $(n, \gamma)$ reaction is small. It is considered that this large self-shielding correction around the upper boundary energy of the unresolved resonance region for the elastic scattering causes the large bump around $100 \mathrm{keV}$ in figure 1.

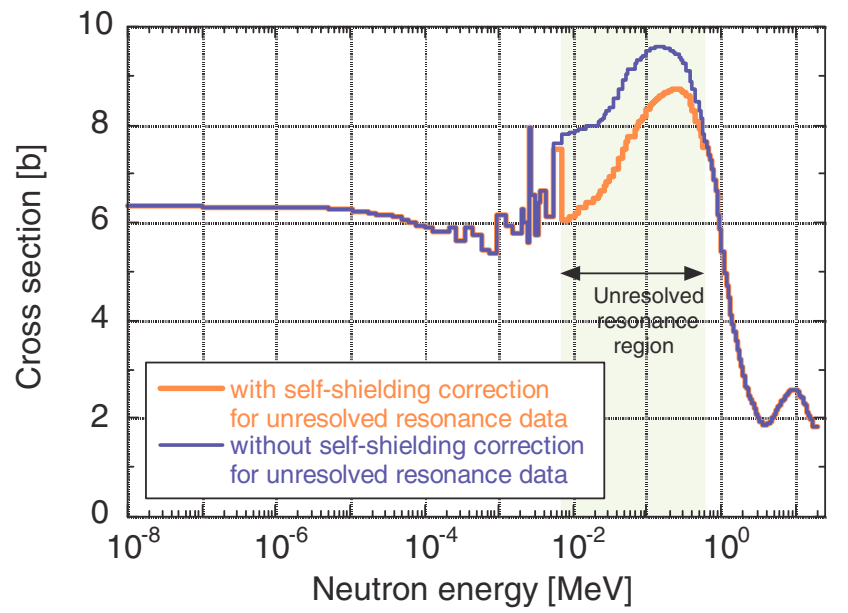

Fig. 4. Self-shielding corrected elastic scattering cross section of temporary ${ }^{93} \mathrm{Nb}$.

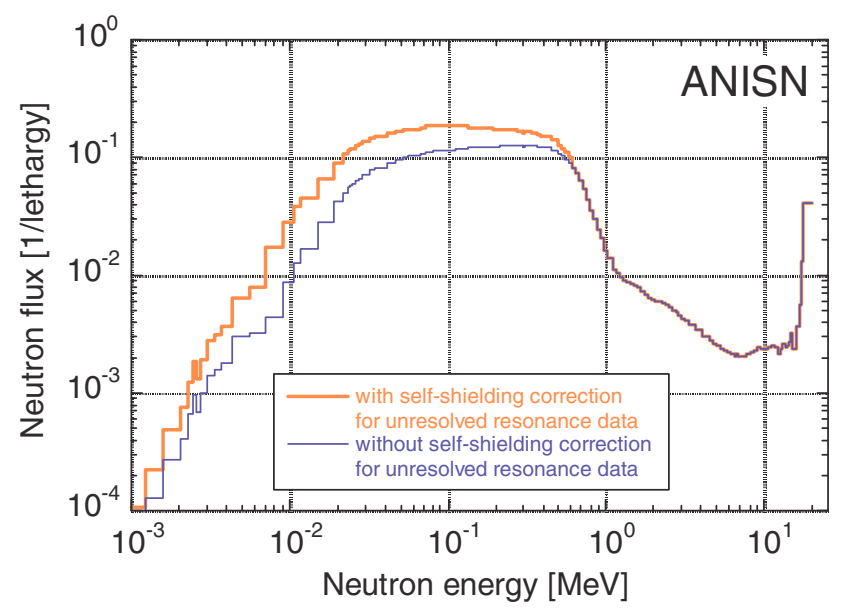

Fig. 5. Calculated leakage neutron spectra from a niobium sphere of $0.5 \mathrm{~m}$ in radius with a $20 \mathrm{MeV}$ neutron in the center by using ANISN and temporary ${ }^{93} \mathrm{Nb}$.

\section{Attempt to revise unresolved resonance data of ${ }^{93} \mathrm{Nb}$ in JENDL-3.3}

Then why is the self-shielding correction too large around the upper boundary energy of the unresolved region of ${ }^{93} \mathrm{Nb}$ in JENDL-3.3? There are two possibilities. One is that the average reduced neutron widths of ${ }^{93} \mathrm{Nb}$ in JENDL-3.3 are too large. But the present average reduced neutron widths are required to reproduce average elastic scattering cross sections in the unresolved resonance region of ${ }^{93} \mathrm{Nb}$ in JENDL-3.3. The other possibility is that the upper boundary energy of the unresolved resonance region is too low. When checking figure 2, it is found that the self-shielding correction tends to decrease with energy. If the upper boundary energy of the unresolved resonance region is increased adequately, the large bump at the upper boundary energy of the unresolved resonance region may decrease. In order to check this idea, we temporarily made a new ${ }^{93} \mathrm{Nb}$ file from the ${ }^{93} \mathrm{Nb}$ file in JENDL-3.3, where the upper boundary energy of the unresolved resonance region is increased from $100 \mathrm{keV}$ to $600 \mathrm{keV}$. Figure 4 shows the self-shielding corrected elastic scattering 


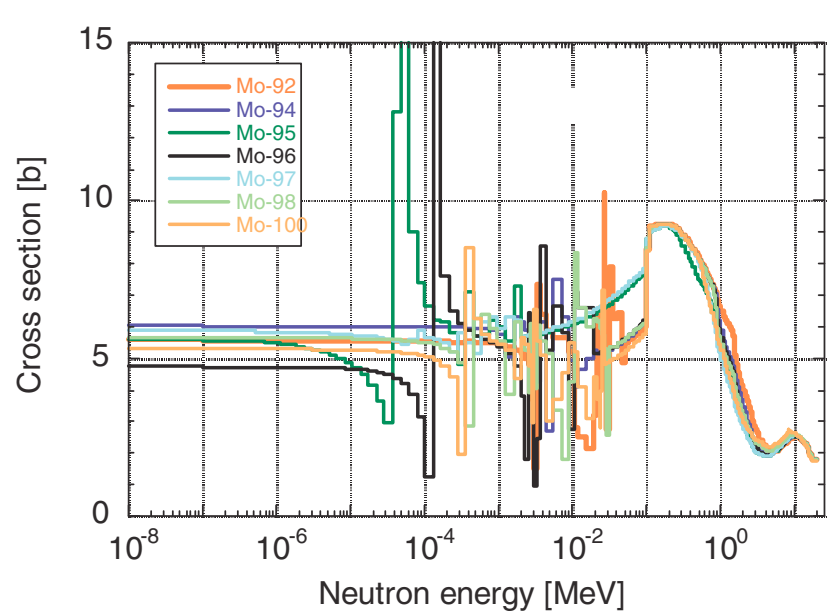

Fig. 6. Self-shielding corrected elastic scattering cross sections of Mo isotopes in JENDL-3.3.

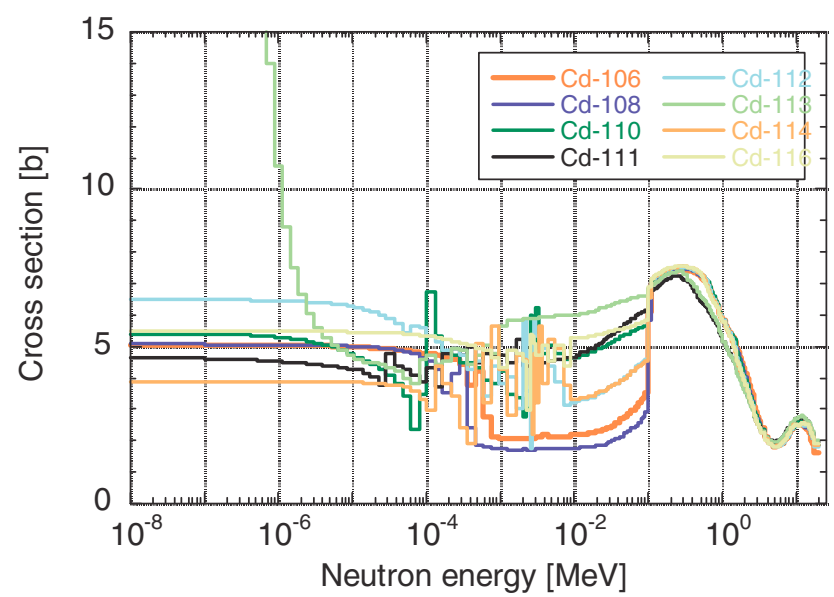

Fig. 7. Self-shielding corrected elastic scattering cross sections of Cd isotopes in JENDL-3.3.

cross section of the new ${ }^{93} \mathrm{Nb}$ file. As expected, the large bump at the upper boundary energy of the unresolved resonance region disappears. By using this revised cross section data, the leakage neutron spectrum from a niobium sphere of $0.5 \mathrm{~m}$ in radius with a $20 \mathrm{MeV}$ neutron in the center has no large strange bump around the upper boundary energy of the unresolved resonance region as shown in figure 5. Experiments such as a leakage neutron spectrum measurement from a niobium sphere of $0.5 \mathrm{~m}$ in radius with DT neutrons in the center are strongly required to confirm whether this attempt is actually right.

\section{Other nuclei with unresolved resonance data in JENDL-3.3}

Do other nuclei with unresolved resonance data in JENDL-3.3 have the same fault as ${ }^{93} \mathrm{Nb}$ ? All the data of nuclei $(210$ nuclei) with unresolved resonance data in JENDL-3.3 were examined. The elastic scattering cross sections with selfshielding correction for each nucleus [100\% abundance] with unresolved resonance data in JENDL-3.3 were deduced with
Table 1. Nuclei with unresolved resonance data in JENDL-3.3.

\begin{tabular}{|c|}
\hline 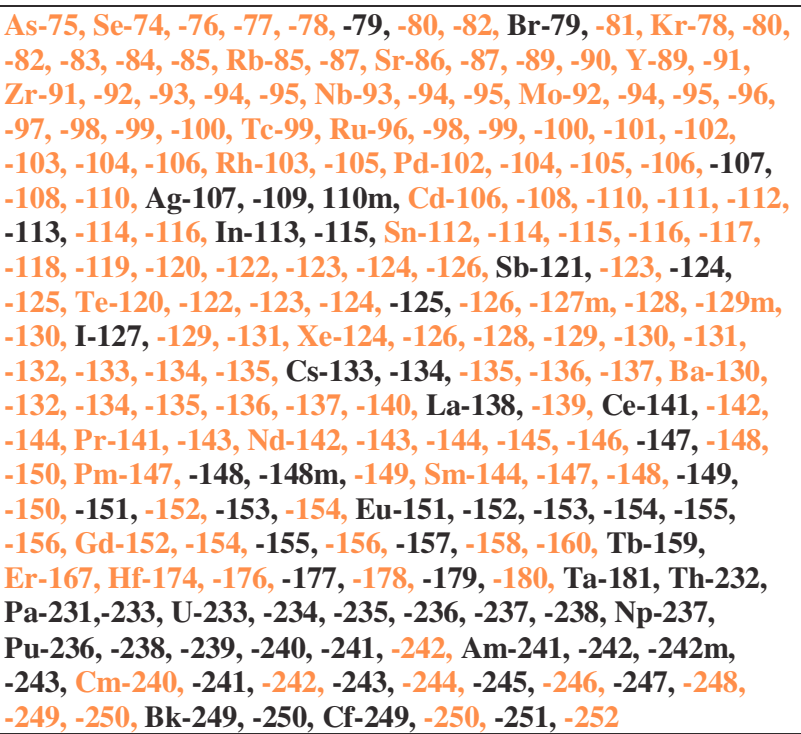 \\
\hline
\end{tabular}

Red: Strange bump appears in the elastic scattering cross section.

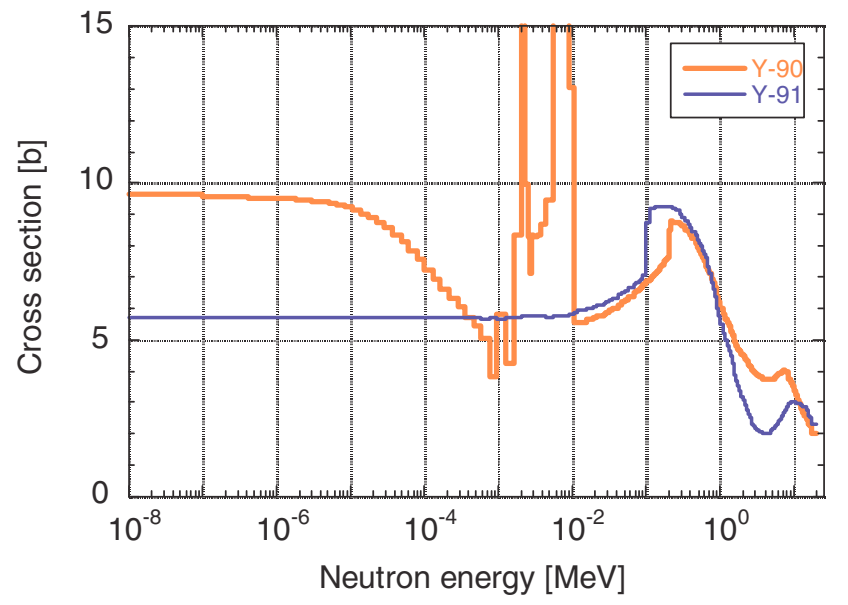

Fig. 8. Self-shielding corrected elastic scattering cross sections of Y isotopes in ENDF/B-VII.0.

TRANSX from MATXSLIB-J33 and plotted. As a result, it is found that elastic scattering cross sections in many nuclei have a similar strange bump at the upper boundary energy of the unresolved resonance region as shown in figures 6 and 7. Table 1 summarizes all the nuclei with unresolved resonance data in JENDL-3.3, where nuclei which may have inadequate unresolved resonance data are indicated in red characters. It is noted that more than half of nuclei with unresolved resonance data in JENDL-3.3 have the same issue as ${ }^{93} \mathrm{Nb}$.

\section{Nuclei with unresolved resonance data in ENDF/B-VII.0}

All the data of nuclei (252 nuclei) with unresolved resonance data in ENDF/B-VII.0 [9] were examined in order to confirm whether other nuclear data libraries have the same issue as 
Table 2. Nuclei with unresolved resonance data in ENDF/B-VII.0.

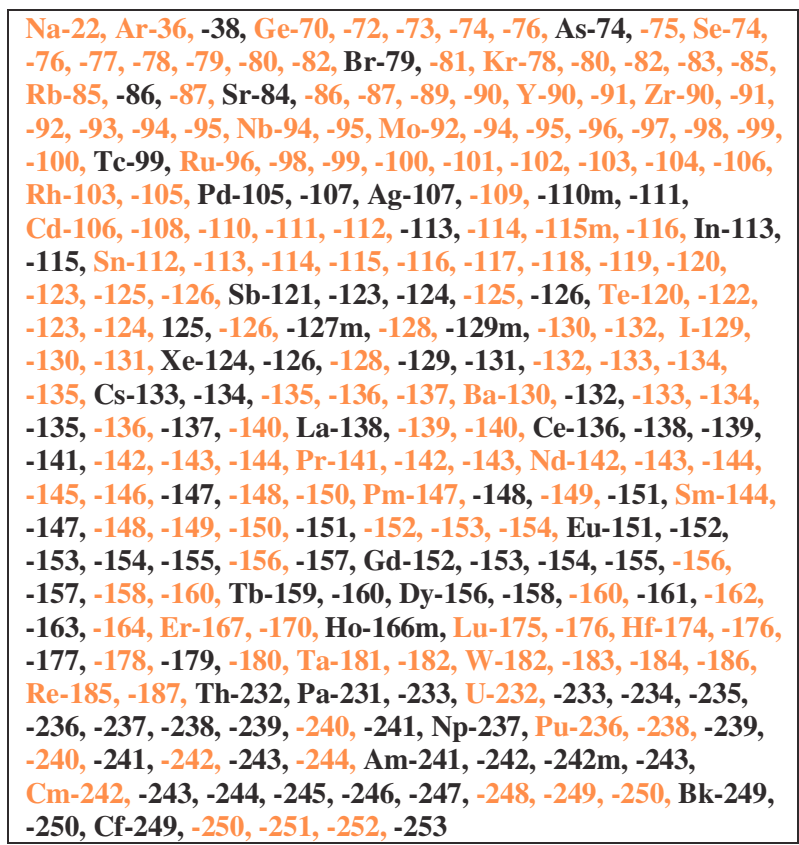

Red: Strange bump appears in the elastic scattering cross section.

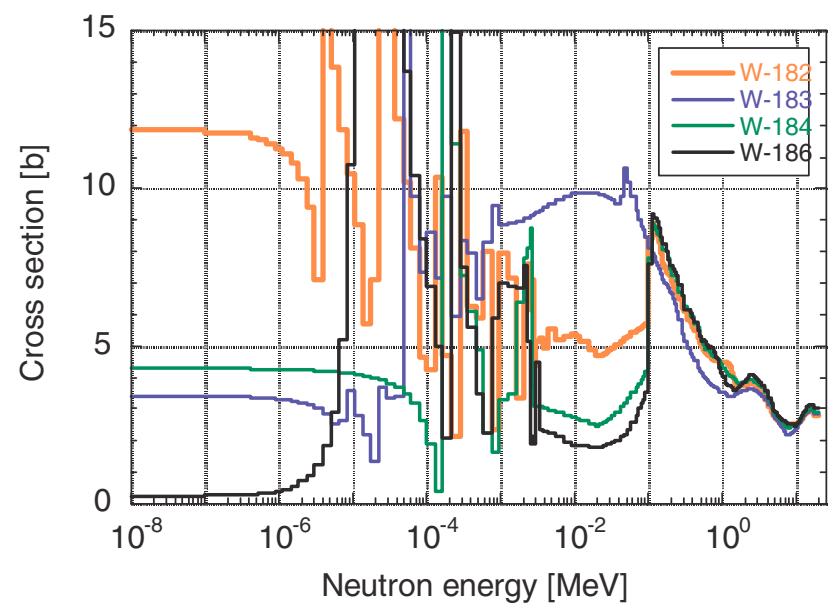

Fig. 9. Self-shielding corrected elastic scattering cross sections of $\mathrm{W}$ isotopes in ENDF/B-VII.0.

JENDL-3.3 for unresolved resonance data. MATXS files of ENDF/B-VII.0 were produced with NJOY99.161. The elastic scattering cross sections with self-shielding correction for each nucleus [100\% abundance] with unresolved resonance data in ENDF/B-VII.0 were deduced with TRANSX from these MATXS files and plotted. As a result, it is found out that the self-shielding correction for elastic scattering cross sections in unresolved resonance region is too large around the upper boundary energy of the unresolved resonance region for more than half of nuclei as shown in figures 8 and 9. Table 2 summarizes all the nuclei with unresolved resonance data in ENDF/B-VII.0. In this table unresolved resonance data of nuclei in red characters may have inadequate unresolved resonance data.

\section{Summary}

It was investigated why a strange bump around $100 \mathrm{keV}$ appeared in calculated leakage neutron spectra from a niobium sphere of $0.5 \mathrm{~m}$ in radius with a $20 \mathrm{MeV}$ neutron in the center. As a result, it is found out that the self-shielding correction for the unresolved resonances is too large around the upper boundary energy of the unresolved resonance region in ${ }^{93} \mathrm{Nb}$ of JENDL-3.3. It is confirmed that this issue can be solved by increasing the upper boundary energy of the unresolved resonance region from $100 \mathrm{keV}$ to $600 \mathrm{keV}$. More than half of nuclei with unresolved resonance data in JENDL-3.3 and ENDF/B-VII.0 have the same issue. It is noted that probably other nuclear data libraries such as JEFF-3.1 [10] also have the same problem for unresolved resonance data. In evaluation for unresolved resonance data, the upper boundary energy of the unresolved resonance region should be set to be high enough by considering self-shielding correction.

\section{References}

1. C. Konno, Y. Ikeda, J. Nucl. Sci. Technol. Suppl. 2, 1037 (2002).

2. DOORS3.2: One, Two- and Three-Dimensional Discrete Ordinates Neutron/Photon Transport Code System, RSICC CODE PACKAGE CCC-650 (1998).

3. J.F. Briesmeister (ed.), LA-13709-M, 2000.

4. K. Shibata et al., J. Nucl. Sci. Technol. 39, 1125 (2002).

5. P. Vontobel, S. Pelloni, EIR-Bericht No. 636, 1987.

6. R.E. MacFarlane, LA-12312-MS, 1993.

7. K. Kosako et al., JAERI-Data/Code 2003-011 (2003).

8. R.E. MacFarlane, D.W. Muir, LA-12740-M, 1994.

9. M.B. Chadwick et al., Nucl. Data Sheets 107, 2931 (2006).

10. A. Koning et al. (ed.), JEFF Report 21, OECD NEA, 2006. 